\title{
Minimally invasive direct repair of lumbar spondylolysis with a pedicle screw and hook construct
}

\author{
Joseph C. Noggle, B.S., ${ }^{1}$ Daniel M. Sciubba, M.D., ${ }^{1}$ Amer F. Samdani, M.D., ${ }^{2}$ \\ D. Greg Anderson, M.D., ${ }^{3}$ RANDal R. Betz, M.D., ${ }^{2}$ And JAhangir Asghar, M.D. ${ }^{2}$
}

${ }^{1}$ Department of Neurosurgery, Johns Hopkins Hospital, Baltimore, Maryland; ${ }^{2}$ Shriners Hospital; and ${ }^{3}$ Rothman Institute, Philadelphia, Pennsylvania

\begin{abstract}
Object. Lumbar spondylolysis occurs in approximately $6 \%$ of the population and presents with localized mechanical back pain, often in young athletes. Surgical treatment may involve decompression, lumbar intersegmental fusion, or direct repair of pars defects. Although such open procedures may effectively resolve symptoms, minimalaccess approaches may additionally decrease collateral damage to soft tissues, allowing young, active patients to resume athletic activities sooner. In this study, the authors review their experience repairing bilateral lumbar spondylolyses with screw and hook constructs placed via a minimal-access approach.

Methods. Five consecutive pediatric patients with bilateral L-5 spondylolysis were treated. Bilateral incisions $(2.5 \mathrm{~cm})$ were made over L-5. Exposure was maintained with bilateral expandable tubular retractor systems. Pedicle screws were placed in the L-5 pedicles and attached to hooks under the L-5 laminae. A direct repair was performed at the pars defect. Clinical characteristics, operative variables, and postoperative outcomes were collected.

Results. All 5 patients underwent surgery; 4 were male (80\%) and 1 was female (20\%), and the mean age was 15.8 years (range 15-17 years). The mean estimated blood loss and duration of surgery were $37 \mathrm{ml}$ (range 15-75 ml) and 1.94 hours (range 1-3 hours), respectively. Postoperative hospital stays ranged from 1 to 3 days (mean 1.8 days). The only complication occurred in 1 patient who experienced minor superficial wound breakdown. All patients have experienced resolution of symptoms at this preliminary stage, which has continued over an 8-month follow-up period.

Conclusions. Lumbar spondylolysis can be adequately and safely treated via minimal-access surgical repair of the pars interarticularis by using pedicle screws and rod-hook constructs. This approach may decrease the collateral soft tissue damage common to open dissections, and may be ideal for young, active surgical candidates.
\end{abstract} (DOI: $10.3171 /$ FOC/2008/25/8/E15)

\section{KEY WORDS - direct surgical repair - minimally invasive surgery • screw and hook construct • spondylolyis}

\section{$\mathrm{L}$} UMBAR spondylolysis classically presents with mechanical back pain, and occurs in approximately $6 \%$ of the population. ${ }^{6,8,12}$ Traditional treatments involve conservative monitoring combined with activity modification or surgical intervention when nonoperative management is not successful. Surgical treatment for the disease can be grouped into 3 major categories, which include lumbar decompression, intersegmental fusion, and the direct repair of pars defects. ${ }^{12}$

Direct surgical repair of pars defects is well documented as an effective treatment in young patients who do not present with instability or severe disc degeneration. ${ }^{1-3,16,20,22}$ Specifically, direct repair may be accomplished with favorable biomechanical and clinical outcomes by techniques including the Buck method and pedicle screw and hook constructs. ${ }^{1-3,7,20}$ In addition, young, active adults are com-

Abbreviation used in this paper: rhBMP- $2=$ recombinant human bone morphogenetic protein-2. mon candidates for such procedures, ${ }^{5,10,12,20}$ and thus may additionally benefit from the avoidance of approach-related damage to back muscles and ligamentous structures that may occur during exposure for standard fusion procedures. For this reason, minimally invasive approaches may be especially relevant.

In this paper we describe the repair of lumbar pars defects in children, which was completed using a minimally invasive approach involving a pedicle screw and laminar hook construct. We highlight the technical aspects of the procedure and clinical outcomes in these patients.

\section{Methods}

\section{Data Collection}

Retrospective review of pediatric patients with spondylolysis was conducted at a single institution over a 1-year period (April 2007-April 2008). Patients treated with sur- 

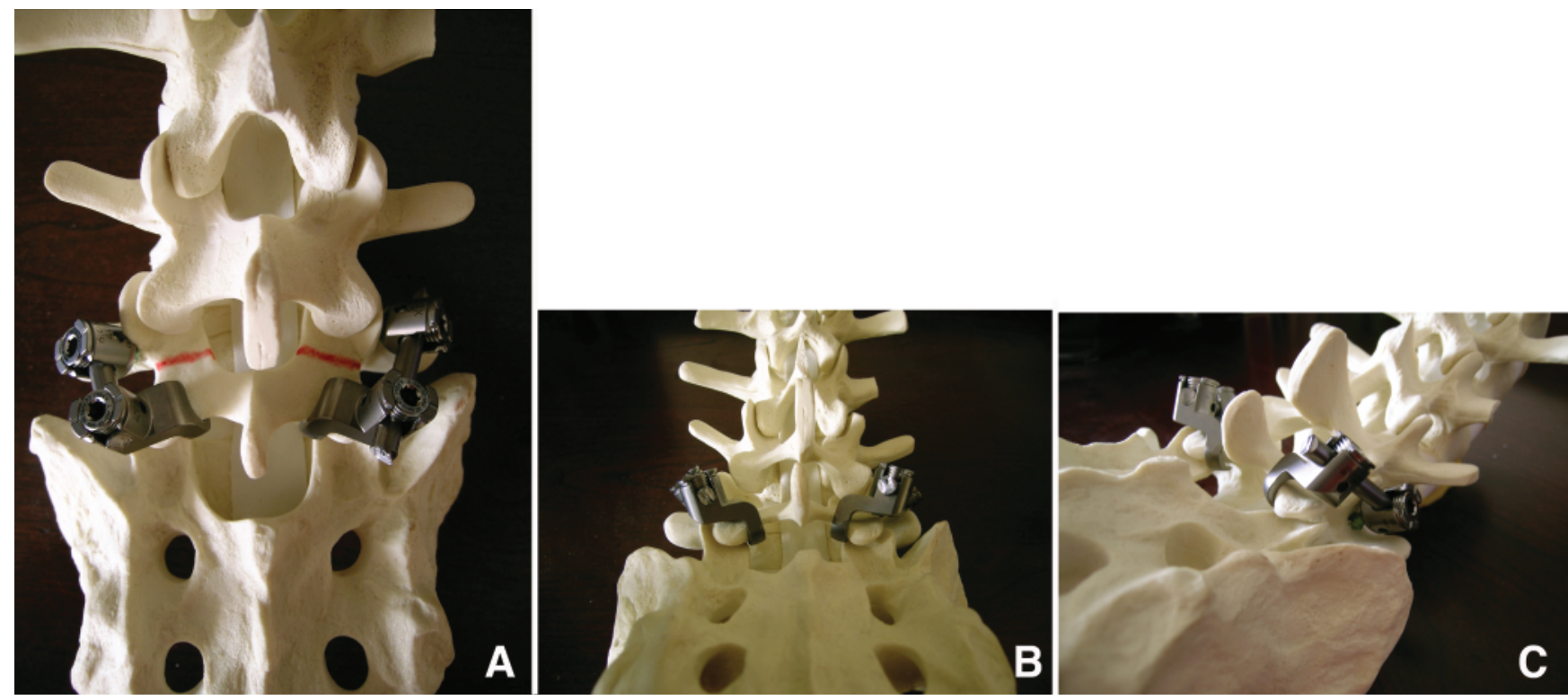

FIG. 1. Photographs of a model of the pedicle screw and laminar hook construct from posterior (A), inferior (B), and right oblique $(\mathrm{C})$ views. Red lines demarcate the bilateral pars interarticularis defects.

gical repair of the pars interarticularis via bilateral pedicle screws and rod and hook constructs were identified and evaluated. Data pertaining to preoperative characteristics, operative management, and postoperative outcomes were collected.

The patients' course consisted of clinical and radiographic evaluation prior to surgery. Operative management involved placement of bilateral pedicle screws and rod and hook constructs via bilateral $2.5-\mathrm{cm}$ skin incisions at L-5 and minimal-access exposure. Pedicle screws were positioned under fluoroscopy by using cannulated screws and Jamshidi needle wires. Offset laminar hooks were then
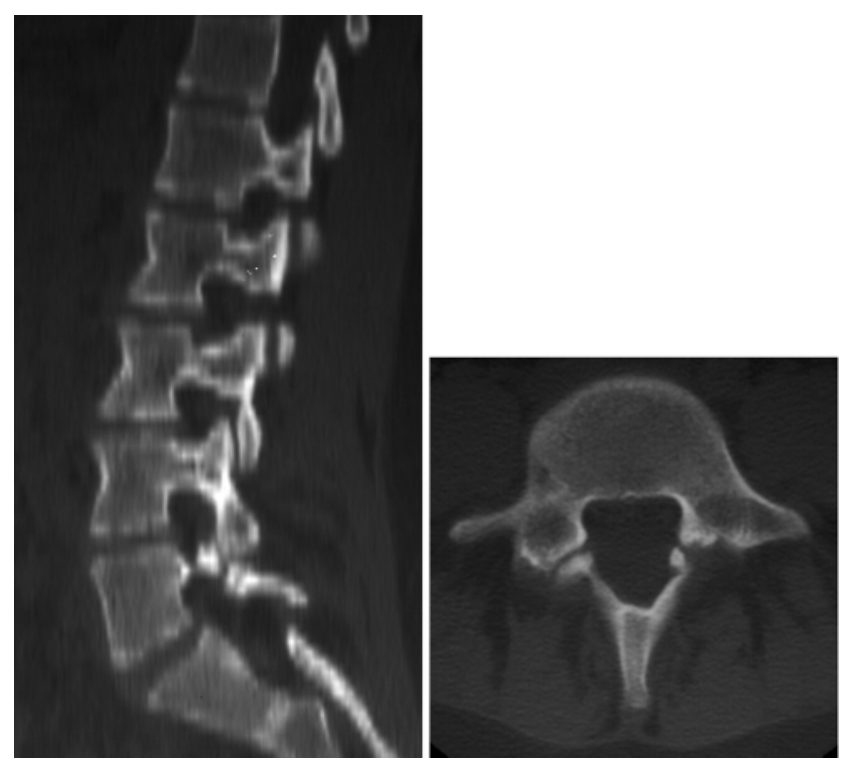

FIG. 2. Illustrative case. Left: Preoperative sagittal CT reconstruction showing spondylolysis at the L5-S1 level. Right: Preoperative axial CT scan showing the pars defect at L-5. placed under the L-5 laminae bilaterally and connected to the L-5 pedicle screws by using rods, which were 3.5-5 $\mathrm{mm}$ in length (Fig. 1). Roughly $2.1 \mathrm{mg}$ of rhBMP-2 (MedtronicSofamor Danek) was placed on each side within the pars defect before closure to facilitate bone repair.

\section{Case Illustration}

History and Examination. This 16-year-old male athlete presented to our clinic with axial low-back pain localized over the L-5 vertebra. The patient had undergone 9 months of nonoperative treatment with minimal resolution of symptoms. After extensive workup and the completion of CT and MR imaging studies, bilateral L-5 spondylolysis (Fig. 2) was diagnosed in this patient. Following informed consent, which included use of rhBMP-2 considered "offlabel" by the Food and Drug Administration, the decision was made to perform surgical repair of the bilateral L-5 pars interarticularis.

Operation. Following intubation, the patient was placed prone on a Jackson table with his knees and hips flexed. Fluoroscopy was used to obtain anteroposterior images of the L-5 vertebral body, and marks were placed on the skin to localize the superior boundary of the L-5 pedicles, the inferior boundary of the L5-S1 disc, and the lateral aspects of the L-5 pedicles.

After sterile preparation and draping, 2 separate $2.5-\mathrm{cm}$ skin incisions were made based at the lateral aspect of the pedicles, from the superior boundary of the L-5 pedicle to the inferior boundary of the L-5 lamina. Cobb elevators and self-retaining retractors were used to expose the fascia, while Bovie cautery and pickups were used to open the fascia widely. Blunt finger dissections were used to split the paraspinal muscles in an avascular plane in a superior-inferior direction, and the transverse processes, facets, and pars were palpated. Initial dilators were docked at the level of the pars and confirmed with fluoroscopy. Subsequent dilators were passed over the initial dilators, and expandable 


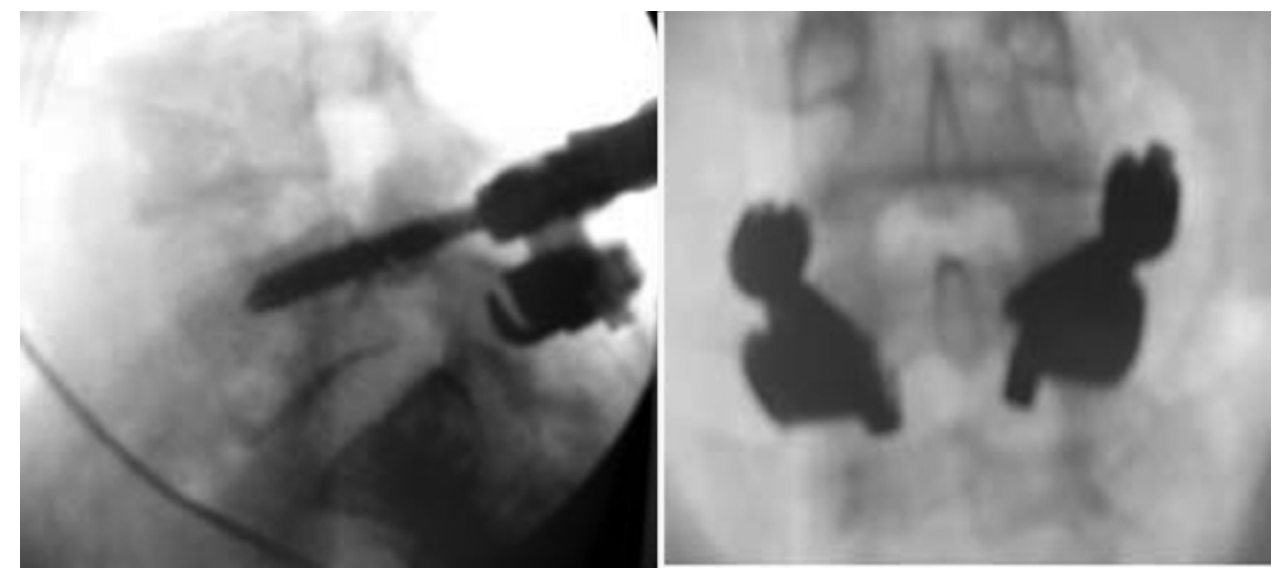

FIG. 3. Illustrative case. Intraoperative fluoroscopic scans of bilateral pars repair at L-5 completed using pedicle screw and hook constructs.

minimal-access retractor systems (Pipeline, DePuy) were placed bilaterally. The remaining muscle was dissected using bipolar cautery to achieve hemostasis, while the facet capsules were carefully preserved. During exposure, the transverse processes, L5-S1 interlaminar windows, L-5

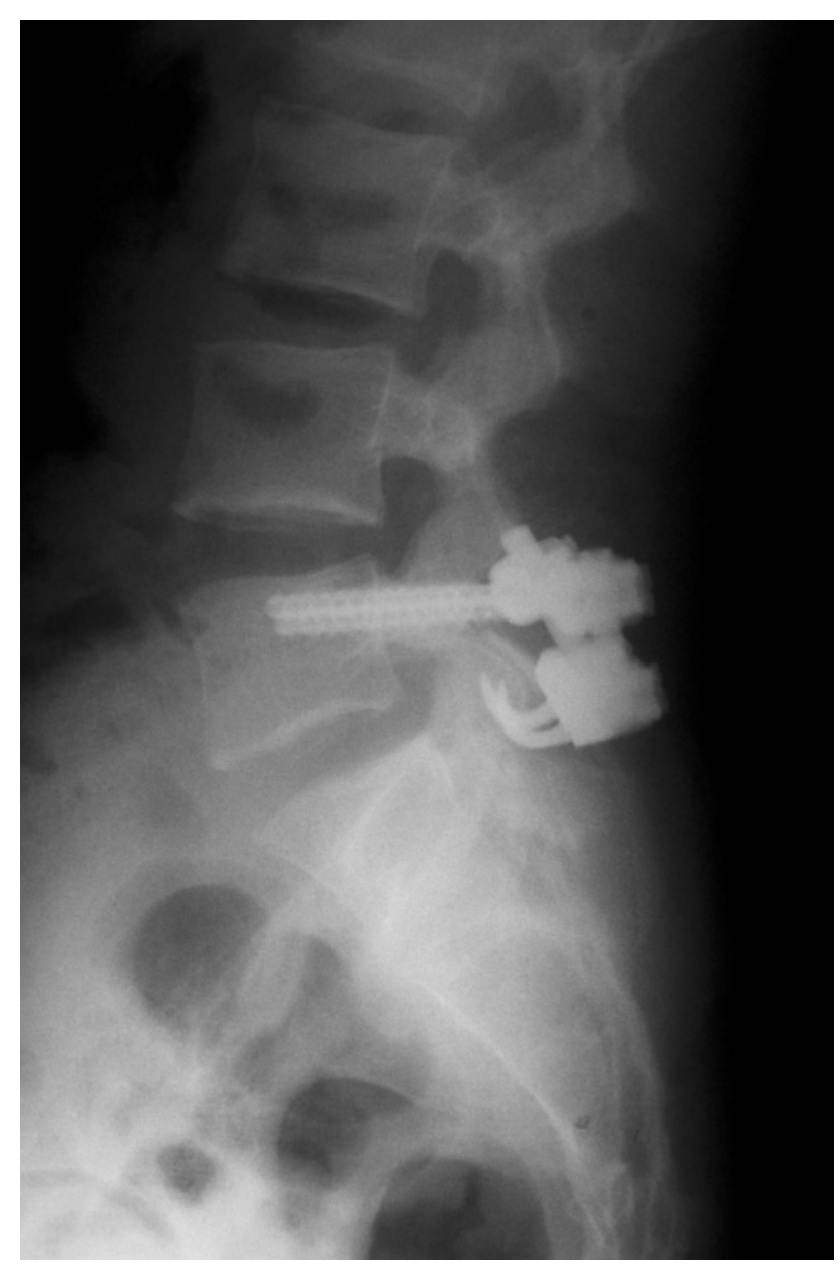

FIG. 4. Illustrative case. Postoperative lateral radiograph showing bilateral pedicle screws and rod and hook constructs at L-5 to correct the pars defect. pars, superior facets, and inferior facets were all successfully identified under direct vision.

Pars defects were located bilaterally at the neck of the superior articular facet at the level of the joint capsule. Fibrous tissue within such defects was scraped out completely in a bilateral fashion, and small troughs were drilled to place rhBMP-2. Bilateral cannulated pedicle screws (7 $\times 50 \mathrm{~mm}$ ) were then placed at L-5 by using Jamshidi needle wires and fluoroscopy for guidance. Laminar hooks connected to short rods were then placed at L-5 (Fig. 3). Once both hooks were seated, the lengths of the attached rods were measured for equality, and a rod holder was used to help drive the rod down into the screws. The laminar hooks and pedicle screw constructs were then compressed and tightened. The wound was then irrigated, and rhBMP2 was placed bilaterally along the pars defects under the rods.

Postoperative Course. The total time of surgery was approximately 3 hours, with an estimated blood loss of 15 $\mathrm{ml}$. The patient displayed no perioperative complications, and spent 2 days in the hospital following surgery. He was released home with the recommendation for outpatient physical therapy and lifting restrictions until 3 months postoperatively. With 6 months of follow-up, his postoperative course has been uneventful, with no major complications and complete resolution of pain (Fig. 4).

\section{Results}

Five pediatric patients were identified, 4 of them male $(80 \%)$ and 1 of them female (20\%), with an average age of 15.8 years (range 15-17 years). The diagnosis in all patients was spondylolysis specific to the L-5 vertebra, and they all presented with axial low-back pain. All patients were symptomatic with the pars defects, to which they were potentially predisposed by their active lifestyles: 2 were farmers (40\%), and 3 were athletes (60\%) (Fig. 5).

All patients were initially treated with nonoperative management for at least 6 months, including physical therapy and activity modification. Eventually, each patient underwent surgical repair of the pars interarticularis with a pedicle screw and rod and hook construct via minimalaccess exposure. The mean intraoperative estimated blood loss and duration of surgery were $37 \mathrm{ml}$ (range 15-75 ml) 

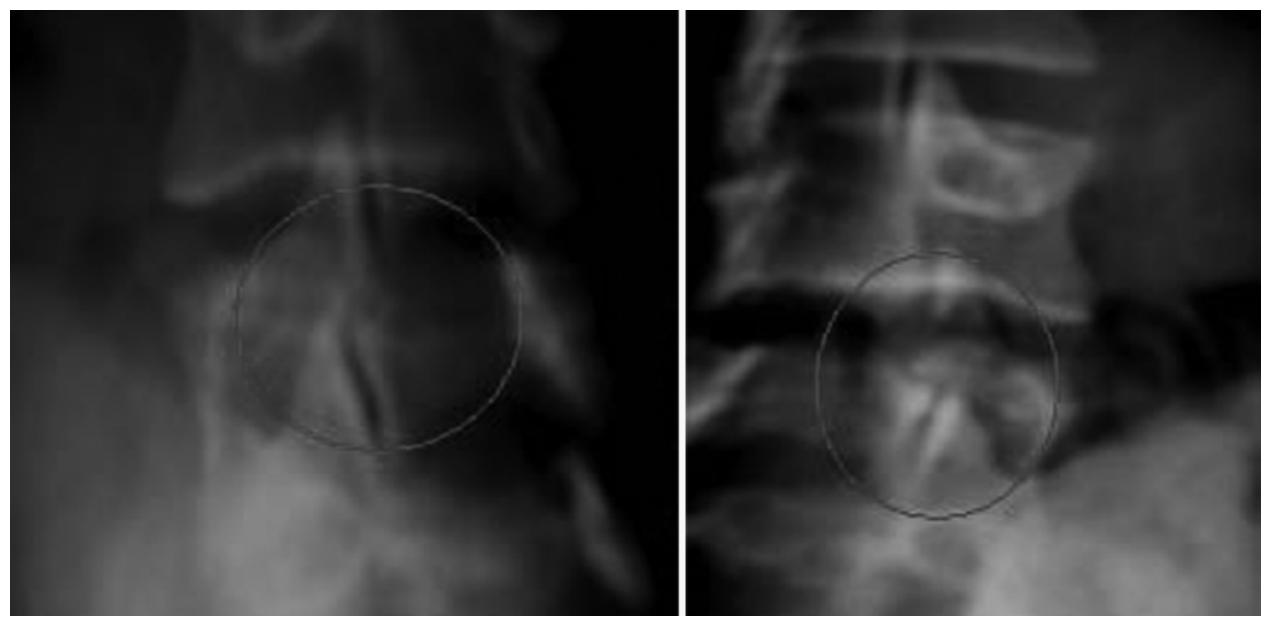

FIG. 5. Illustrative case. Preoperative oblique radiographs obtained in a 16 -year-old boy with bilateral L-5 spondylolytic defects (circled areas). This patient previously had demonstrated increased radionuclide uptake on bone scans bilaterally at L-5, and continued to be symptomatic despite 2 years of nonoperative treatment, including extensive bracing.

and 1.94 hours (range 1-3 hours), respectively. Postoperative hospital stays ranged from 1 to 3 days (mean 1.8 days).

All patients were followed for $>6$ months (mean 7.2 months, range 6-9 months). Four patients (80\%) were treated postoperatively with physical therapy and removed from all activity restrictions after 1 month. One patient (20\%) chose not to attend physical therapy and was removed from all activity restrictions after 3 months. No patient suffered major complications; however, 1 patient (20\%) experienced minor superficial wound breakdown. All patients experienced resolution of symptoms with treatment (Table 1). In addition, all patients have demonstrated fusion on CT scans obtained at 6 months after surgery (Fig. 6).

\section{TABLE 1}

Demographic data in 230 patients with degenerative lumbar spine disease*

\begin{tabular}{|c|c|c|c|c|}
\hline \multirow[b]{2}{*}{ Factor } & \multicolumn{2}{|c|}{ LMD } & \multicolumn{2}{|c|}{ Laminectomy } \\
\hline & 1-Level & 2-Level & 1-Level & 2-Level \\
\hline no. of patients & 141 & 14 & 49 & 26 \\
\hline $\operatorname{sex}(M / F)$ & $75 / 66$ & $9 / 5$ & $26 / 23$ & $15 / 11$ \\
\hline age in yrs & & & & \\
\hline mean $\pm \mathrm{SD}$ & $48.3 \pm 13.1$ & $43.4 \pm 13.9$ & $58.0 \pm 15.7$ & $66.0 \pm 13.7$ \\
\hline $\begin{array}{l}\text { range } \\
\text { disc level }(\%)\end{array}$ & $22-85$ & $18-65$ & $28-90$ & $19-82$ \\
\hline L2-3 & $2(1.4)$ & NA & $1(2.0)$ & NA \\
\hline L3-4 & $8(5.6)$ & NA & $10(20.4)$ & NA \\
\hline L4-5 & $55(39)$ & NA & $30(61.2)$ & NA \\
\hline L5-S1 & $76(54)$ & NA & $8(16.4)$ & NA \\
\hline L2-3, L3-4 & $\mathrm{NA}$ & 0 & NA & $2(7.7)$ \\
\hline L3-4, L4-5 & NA & $1(7.1)$ & NA & $11(42.3)$ \\
\hline L4-5, L5-S1 & NA & $13(92.9)$ & NA & $12(46.1)$ \\
\hline L4-5, L1-2 & NA & NA & NA & $1(3.9)$ \\
\hline
\end{tabular}

* NA = not applicable; $\mathrm{SD}=$ standard deviation.

\section{Discussion}

Pars defects are not uncommon in the population, presenting with symptoms of mechanical back pain, the majority of which can be managed conservatively., ${ }^{6,8,12} \mathrm{Al}-$ though numerous techniques have been described to address such disorders surgically, minimal-access procedures may have the additional benefit of decreasing collateral damage to soft tissues, notably muscle, allowing young, active patients to resume athletic activities. In this manuscript, we describe the technique of repairing bilateral lumbar pars defects with screw and hook constructs that are placed via minimal-access retractor systems.

In this series, 5 patients with L-5 spondylolysis underwent surgical repair of the pars interarticularis via bilateral pedicle screws and rod and hook constructs. The average length of surgery was 1.94 hours, estimated blood loss was $37 \mathrm{ml}$, and the average length of hospital stay was 1.8 days. Four patients were allowed to return to normal activity after 1 month, and the other patient did after 3 months. At last follow-up evaluation, all patients demonstrated complete resolution of symptoms.

Despite the fact that conservative measures (rest, bracing, physical therapy, restriction of activity, pain medication) are successful for most patients with lumbar pars defects, surgical treatments are indicated for those patients who do not respond. Internal fixation was first proposed by Buck $^{2}$ in 1970, and he described direct repair by placing a screw through the pars interarticularis. ${ }^{3}$ Due to complications with healing of the defect and hardware failure, Nicol and $\mathrm{Scott}^{16}$ proposed a wiring technique to stabilize the posterior arch, which has led to an increased rate of fusion. ${ }^{6}$ In 1984, as described by multiple authors, ,9,14,18,22 Morsher introduced the use of the laminar hook to allow compression of the posterior arch and bone graft, and evolution of his original concept later involved use of segmental pedicle screw and hook fixation.

This current concept of pedicle screw and hook fixation is beneficial in that it is extremely rigid and thus obviates the need for postoperative immobilization. Deguchi et al. ${ }^{7}$ have demonstrated biomechanically that such a construct 


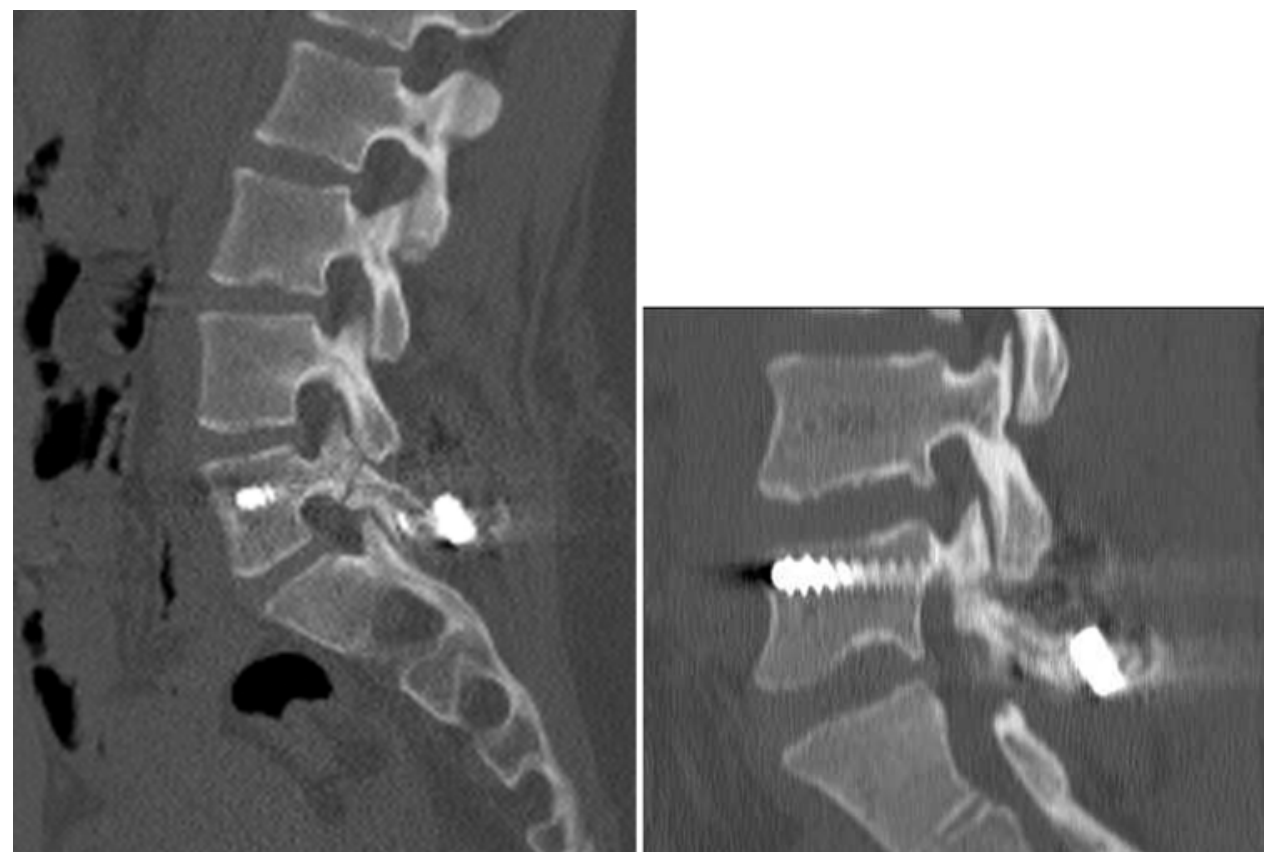

FIG. 6. Sagittal CT reconstruction images obtained in another patient in the cohort immediately postoperatively (left) and at the 6-month follow-up visit (right), showing evolution of bone healing at the pars interarticularis defect.

minimizes micromotion across the defect. Second, pedicle screw insertion, unlike the placement of pars interarticularis screws, is a familiar technique to most surgeons. Finally, by avoiding segmental instrumentation, no loss of lumbar motion segments occurs, as happens with fusion procedures.

However, despite the potential benefits, open placement of such systems may be associated with collateral damage to soft tissue..$^{11,12}$ In patients undergoing repeat posterior lumbar surgery via standard dissection, Kawaguchi et al..$^{15}$ observed late back muscle injury $>10$ months after the original operation. Following histological and histochemical evaluation of the tissue, they concluded that increased retraction time and pressure were related to enhanced muscle damage. Various authors have sought to reduce approach-related injury in lumbar spine procedures through minimally invasive, muscle-splitting approaches. ${ }^{12,13,19,21}$ Patients undergoing such procedures have demonstrated improved functional outcomes. For example, Rahman et al. ${ }^{17}$ report earlier postoperative mobility in patients experiencing minimally invasive versus open lumbar laminectomies. Chao et al. ${ }^{4}$ have sought to corroborate such data by demonstrating worse postoperative trauma resulting from open procedures. Specifically, high concentrations of interleukin-6, C-reactive protein, and creatine kinase were obtained in blood samples of patients undergoing open versus minimal-access surgeries.

\section{Conclusions}

In young, active patients in whom nonoperative treatment for lumbar spondylolysis fails, direct repair of the pars defect with screw and hook construct can provide significant relief without the need for segmental fusion. With use of minimal-access approaches, additional benefit may result from decreased tissue dissection, reduced blood loss, and shorter recovery time.

\section{Acknowledgments}

We thank Kevin Moroney and Keith Smith for their help in preparing the images in this manuscript.

\section{References}

1. Bonnici AV, Koka SR, Richards DJ: Results of Buck screw fusion in grade I spondylolisthesis. J R Soc Med 84:270-273, 1991

2. Buck JE: Direct repair of the defect in spondylolisthesis. Preliminary report. J Bone Joint Surg Br 52:432-437, 1970

3. Buck JE: Further thoughts on direct repair of the defect in spondylolisthesis. J Bone Joint Surg Br 61:123, 1979

4. Chao Z, Yue Z, Tong-wei C, Jian W, Yong H, Yong P: Microendoscopic discectomy, a less traumatic procedure for lumbar disk herniation. Chin J Traumatol 10:311-314, 2007

5. Debnath UK, Freeman BJ, Gregory P, de la Harpe D, Kerslake RW, Webb JK: Clinical outcome and return to sport after the surgical treatment of spondylolysis in young athletes. J Bone Joint Surg Br 85:244-249, 2003

6. Debusscher F, Troussel S: Direct repair of defects in lumbar spondylolysis with a new pedicle screw hook fixation: clinical, functional and CT-assessed study. Eur Spine J 16:1650-1658, 2007

7. Deguchi M, Rapoff AJ, Zdeblick TA: Biomechanical comparison of spondylolysis fixation techniques. Spine 24:328-333, 1999

8. Fredrickson BE, Baker D, McHolick WJ, Yuan HA, Lubicky JP: The natural history of spondylolysis and spondylolisthesis. J Bone Joint Surg Am 66:699-707, 1984

9. Gillet P, Petit M: Direct repair of spondylolysis without spondylolisthesis, using a rod-screw construct and bone grafting of the pars defect. Spine 24:1252-1256, 1999

10. Hardcastle PH: Repair of spondylolysis in young fast bowlers. J Bone Joint Surg Br 75:398-402, 1993

11. Hensinger RN: Spondylolysis and spondylolisthesis in children and adolescents. J Bone Joint Surg Am 71:1098-1107, 1989 
12. Higashino K, Sairyo K, Katoh S, Sakai T, Kosaka H, Yasui N: Minimally invasive technique for direct repair of the pars defects in young adults using a spinal endoscope: a technical note. Minim Invasive Neurosurg 50:182-186, 2007

13. Huang TJ, Hsu RW, Li YY, Cheng CC: Less systemic cytokine response in patients following microendoscopic versus open lumbar discectomy. J Orthop Res 23:406-411, 2005

14. Kakiuchi M: Repair of the defect in spondylolysis. Durable fixation with pedicle screws and laminar hooks. J Bone Joint Surg Am 79:818-825, 1997

15. Kawaguchi Y, Matsui H, Tsuji H: Back muscle injury after posterior lumbar spine surgery. Part 2: Histologic and histochemical analyses in humans. Spine 19:2598-2602, 1994

16. Nicol RO, Scott JH: Lytic spondylolysis. Repair by wiring. Spine 11:1027-1030, 1986

17. Rahman M, Summers LE, Richter B, Mimran RI, Jacob RP: Comparison of techniques for decompressive lumbar laminectomy: the minimally invasive versus the "classic" open approach. Minim Invasive Neurosurg 51:100-105, 2008

18. Roca J, Iborra M, Cavanilles-Walker JM, Albertí G: Direct repair of spondylolysis using a new pedicle screw hook fixation: clinical and CT-assessed study: an analysis of 19 patients. J Spinal Disord Tech 18 Suppl: S82-S89, 2005
19. Sairyo K, Goel VK, Masuda A, Biyani A, Ebraheim N, Mishiro T, et al: Biomechanical rationale of endoscopic decompression for lumbar spondylolysis as an effective minimally invasive procedure - a study based on the finite element analysis. Minim Invasive Neurosurg 48:119-122, 2005

20. Sairyo K, Katoh S, Sakamaki T, Komatsubara S, Endo K, Yasui $\mathrm{N}$ : Three successive stress fractures at the same vertebral level in an adolescent baseball player. Am J Sports Med 31:606-610, 2003

21. Sasaoka R, Nakamura H, Konishi S, Nagayama R, Suzuki E, Terai $\mathrm{H}$, et al: Objective assessment of reduced invasiveness in MED. Compared with conventional one-level laminotomy. Eur Spine J 15:577-582, 2006

22. Tokuhashi Y, Matsuzaki H: Repair of defects in spondylolysis by segmental pedicular screw hook fixation. A preliminary report. Spine 21:2041-2045, 1996

Address correspondence to: Daniel M. Sciubba, M.D., 600 North Wolfe Street, Meyer 8-161, Baltimore, Maryland 21287. email: dsciubb1@jhmi.edu. 